\title{
THE MILLIARCSECOND STRUCTURE OF ACTIVE GALACTIC NUCLEI OBSERVED WITH VLBI
}

\author{
T. J. PEARSON and A. C. S. READHEAD \\ Owens Valley Radio Observatory \\ California Institute of Technology \\ Pasadena, California 91125 \\ $U S A$
}

\section{A VLBI Survey}

Very Long Baseline Interferometry at radio wavelengths is the only technique available for imaging the central few parsecs of powerful radio galaxies and quasars. VLBI observations have shown that in many nuclei radio-emitting material is collimated into a jet on a scale less than a parsec and ejected at relativistic velocities. The interpretation of the observations is complicated by the relativistic motion, however: the images are dominated by those parts of the source that are moving almost directly towards the observer, and thus amplified by relativistic aberration. Nonetheless, the VLBI images are vital for understanding the nature of the central engine, the cause of the collimation, and the physics of the jets.

VLBI imaging first became possible with the exploitation of 'closure phase' information in 1977. Since that time, a large number of active nuclei have been imaged at several wavelengths, many of them repeatedly. These observations have provided detailed documentation of the evolution of individual sources, notably the well-known superluminal sources $3 \mathrm{C} 273,3 \mathrm{C} 345$, and $3 \mathrm{C} 120$. To supplement these studies of individual sources, to determine the full range of morphologies exhibited, and to provide a large sample for possible statistical analysis, we have made $5 \mathrm{GHz}$ images of the 65 brightest radio sources at $5 \mathrm{GHz}$ north of declination $35^{\circ}$. The observations have a resolution of about 1 milliarcsec and a dynamic range of $\gtrsim 100: 1$. First-epoch results have been published (Pearson and Readhead 1988) and we have completed observations at a second and a third epoch in collaboration with our colleagues Peter Barthel and Steve Myers.

\section{Morphological Classification}

Without greater understanding of the physics of active nuclei, it is premature to attempt a physical classification of the sources. It is, however, possible to make a morphological classification which may prove to have some physical significance. Our classification is described in detail by Pearson and Readhead (1988). It is based on the morphology of the dominant radio component, the radio spectrum, and the nature of the extended radio emission. There are five major classes: (1) Very compact, compact, and asymmetric objects [25 objects]. These range from virtually unresolved sources ( $>90 \%$ of flux in a component 
$<1$ mas) to well-resolved objects with a flat-spectrum, compact core and a one-sided, steep-spectrum, elongated jet. No two-sided jets are seen. The sources can be arranged in a regular progression in which the jet becomes increasingly prominent. The cores are highly variable; the jets are more stable and more strongly polarized. All are identified with quasars or BL Lac objects. (2) Compact double objects [7], dominated by two components of almost equal flux density. This class appears to confuse two sub-classes, which can be distinguished by spectrum. Those with steep high-frequency spectra are a distinct class, first identified by Phillips and Mutel (1982; see also Hodges and Mutel 1987). They differ sharply from the 'core-jet' sources in morphology, in their low polarization and low variability, and in having no detectable kiloparsec-scale emission, and they are identified with galaxies rather than quasars or BL Lac objects. The objects with flat high-frequency spectra also show low variability but are identified with quasars. (3) Irregular flat spectrum objects [2], a miscellaneous class which includes NGC 1275. (4) Steep-spectrum compact objects [7]. These objects show structure on all angular scales from 1 mas to 1 arcsec, with complex, jet-like features. It has been suggested that in these objects the structure is largely due to interaction of a jet or jets with the interstellar medium. (5) Lobe-dominated objects [19]. Almost all of the lobe-dominated objects have detectable radio cores, but most are two weak for imaging with narrow-bandwidth VLBI. The exceptions show elongated jet-like structures aligned with the large-scale structure.

\section{Superluminal Motion}

Superluminal motion is common: in the sample of 65 sources, at least nine are superluminal. We have discovered four of these by comparison of our first- and second-epoch images, and we expect to find more from a careful analysis of the third-epoch images. We can only make a reliable measurement of internal proper motion in sources which show two or more well-separated peaks of emission, and many of the sources are uncoöperative. Superluminal motion is not confined to the 'core-jet' class, although $6 / 25$ of these sources are superluminal. Superluminal motion is also seen in a lobe-dominated source (3C 179: Porcas 1987), a steep-spectrum compact source (3C 216: Barthel et al. 1988), and a compact double with flat high-frequency spectrum (4C 39.25: Shaffer et al. 1987). This last source is interesting as it shows superluminal motion of a component between two stationary components. The number of superluminal sources is now high enough to permit statistical study (Cohen et al. 1988).

So far we have found only two sources $(0710+439$ and $2021+614)$ where we can place definitive subluminal limits on the component proper motion. Both of these are compact doubles, again distinguishing this class from the 'core-jet' sources.

\section{Beaming and Unified Theories}

The ubiquity of apparent superluminal motion confirms that something is moving relativistically in the core-dominated radio nuclei. Additional evidence comes from the high brightness temperatures and the absence of synchrotron self-Compton X-radiation. In the 'unified theory' of extragalactic radio sources (e.g., Readhead et al. 1978; Orr and Browne 1982), the core-dominated sources are supposed to be intrinsically similar to the lobe-dominated sources, but with the core amplified by relativistic beaming. This idea 
naturally explains many of the properties of the core-dominated sources. Further support is provided by high dynamic-range VLA images of three of the newly-discovered superluminal sources, which show an extended kiloparsec structure with a jet (on the same side of the nucleus as the parsec-scale jet) feeding a lobe on one side, and a similar lobe (but no jet) on the other side. The unified theory implies that there should be a large population of unbeamed sources. Orr and Browne suggested that this population consists of the extended, lobe-dominated quasars. There are some difficulties with this picture; in particular, if relativistic beaming only occurs along the source axis, then core-dominated, superluminal quasars (which are seen end-on) should have smaller angular sizes than the lobe-dominated quasars; this is not generally true, and indeed the largest known quasar is superluminal (Barthel 1987; see also Simon et al. 1987).

It is possible that this problem can be solved by postulating that the unbeamed population consists of radio galaxies rather than quasars; i.e., whether a nucleus appears as a radio galaxy or a quasar depends on its orientation relative to the observer (see, e.g., the discussion by Browne and Scheuer in Zensus and Pearson 1987). Recently Barthel (1988) has shown that the angular size statistics of the 3CR sources (presumed to be an orientation-unbiased sample) are consistent with the idea that an object appears as a quasar only within a cone of half-angle $\sim 45^{\circ}$. The cause of this anisotropy in the optical radiation is not determined, but it is presumably due to some combination of relativistic beaming and obscuration. There is not space here to discuss the implications of this hypothesis, or the supporting evidence from optical spectra and polarization (e.g., Antonucci 1984; Antonucci and Miller 1985; Krolik and Begelman 1986), vut doubtless they will be addressed in many other contributions to this Symposium.

This work was supported in part by the National Science Foundation under grant AST 85-09822.

\section{References}

Antonucci, R. R. J. 1984, Astrophys. J., 278, 499.

Antonucci, R. R. J., and Miller, J. S. 1985, Astrophys. J., 297, 621.

Barthel, P. D. 1987, in Zensus and Pearson 1987, p. 148.

Barthel, P. D. 1988, Astrophys. J., in press.

Barthel, P. D., Pearson, T. J., and Readhead, A. C. S. 1988, Astrophys. J. (Letters), 329, L51.

Cohen, M. H., Barthel, P. D., Pearson, T. J., and Zensus, J. A. 1988, Astrophys. J., 329, 1.

Hodges, M. W., and Mutel, R. L. 1987, in Zensus and Pearson 1987, p. 168.

Krolik, J. H., and Begelman, M. C. 1986, Astrophys. J. (Letters), 308, L55.

Orr, M. J. L., and Browne, I. W. A. 1982, Monthly Notices Roy. Astron. Soc., 200, 1067.

Pearson, T. J., and Readhead, A. C. S. 1988, Astrophys. J., 328, 114.

Phillips, R. B., and Mutel, R. L. 1982, Astron. Astrophys., 106, 21.

Porcas, R. W. 1987, in Zensus and Pearson 1987, p. 12.

Readhead, A. C. S., Cohen, M. H., Pearson, T. J., and Wilkinson, P. N. 1978, Nature, 276, 768.

Shaffer, D. B., Marscher, A. P., Marcaide, J., and Romney, J. D. 1987, Astrophys. J. (Letters), 314, L1.

Simon, R. S. et al. 1987, in Zensus and Pearson 1987, p. 155.

Zensus, J. A., and Pearson, T. J. (eds.) 1987, Superluminal Radio Sources (Cambridge: Cambridge University Press). 


\section{DISCUSSION}

BIERMANN Relativistic bulk motion can be argued for on the basis of variability, a Compton discrepancy, or apparent superluminal motion. My colleagues at Bonn and I studied a complete sample (13 sources) of flat-spectrum radio sources at many epochs and many frequencies with the VLBI, for variability and in X-rays (up to 5 epochs). In 12 out of 13 sources, we find evidence for relativistic effects, thus strongly supporting the standard picture. 